\title{
On pseudo-complex bases for monogenic polynomials
}

\author{
C. Cruz ${ }^{*}$ M. I. Falcão*,† and H. R. Malonek ${ }^{*, * *}$ \\ ${ }^{*}$ Center for Research and Development in Mathematics and Applications, University of Aveiro, Portugal \\ ${ }^{\dagger}$ Department of Mathematics and Applications, University of Minho, Portugal \\ ${ }^{* *}$ Department of Mathematics, University of Aveiro, Portugal
}

\begin{abstract}
In the recent past, the problem of constructing bases for spaces of monogenic polynomials, in the framework of Clifford Analysis, has been considered by several authors, using different methods. In this talk we consider bases of 3D monogenic polynomials isomorphic to the complex powers, which are particularly easy to handle, from the computational point of view. Explicit constructions of such polynomial bases are performed and a numerical cost comparison with the well known Fueter polynomial basis is carried out.
\end{abstract}

Keywords: homogeneous monogenic polynomials, hypercomplex analysis, Appell sets

PACS: 02.30.-f, 02.30.Lt

\section{INTRODUCTION AND BASIC NOTATION}

Let $\left\{e_{1}, e_{2}, \cdots, e_{n}\right\}$ be an orthonormal base of the Euclidean vector space $\mathbb{R}^{n}$ with a product according to the multiplication rules

$$
e_{k} e_{l}+e_{l} e_{k}=-2 \delta_{k l}, k, l=1, \ldots, n,
$$

where $\delta_{k l}$ is the Kronecker symbol. This noncommutative product generates the $2^{n}$-dimensional Clifford algebra $C \ell_{0, n}$ over $\mathbb{R}$ and the set $\left\{e_{A}: A \subseteq\right.$ $\{1, \cdots, n\}\}$ with $e_{A}=e_{h_{1}} e_{h_{2}} \cdots e_{h_{r}}, 1 \leq h_{1}<\cdots<h_{r} \leq$ $n, e_{\emptyset}=e_{0}=1$, forms a basis of $C \ell_{0, n}$. Considering the subset

$$
\mathscr{A}_{n}:=\operatorname{span}_{\mathbb{R}}\left\{1, e_{1}, \ldots, e_{n}\right\} \subset C \ell_{0, n},
$$

the real vector space $\mathbb{R}^{n+1}$ can be embedded in $\mathscr{A}_{n}$ by the identification of each element $\left(x_{0}, x_{1}, \cdots, x_{n}\right) \in \mathbb{R}^{n+1}$ with the paravector $x \in \mathscr{A}_{n}$. Here, $x_{0}$ and $\underline{x}=e_{1} x_{1}+$ $\cdots+e_{n} x_{n}$ are, the so-called, scalar and vector parts of the paravector $x \in \mathscr{A}_{n}$. The conjugate of $x$ is $\bar{x}=x_{0}-\underline{x}$ and the norm $|x|$ of $x$ is defined by $|x|=\sqrt{x \bar{x}}$.

In what follows we consider $C l_{0, n}$-valued functions defined in some open subset $\Omega \subset \mathbb{R}^{n+1}$, i.e. functions of the form $f(z)=\sum_{A} f_{A}(z) e_{A}$, where $f_{A}(z)$ are real valued. The generalized Cauchy-Riemann operator in $\mathbb{R}^{n+1}, n \geq 1$, is defined by

$$
\bar{\partial}:=\frac{1}{2}\left(\partial_{0}+\partial_{\underline{x}}\right),
$$

where

$$
\partial_{0}:=\frac{\partial}{\partial x_{0}}, \quad \text { and } \quad \partial_{\underline{x}}:=e_{1} \frac{\partial}{\partial x_{1}}+\cdots+e_{n} \frac{\partial}{\partial x_{n}} .
$$

Functions $f$ satisfying $\bar{\partial} f=0$ (resp. $f \bar{\partial}=0)$ are called left monogenic (resp. right monogenic). Following [1], we use $f^{\prime}=\partial f=\frac{1}{2}\left(\partial_{0}-\partial_{x}\right)$ as the hypercomplex derivative of a monogenic function $f$. Since a hypercomplex differentiable function belongs to the kernel of $\bar{\partial}$, it follows that in fact $f^{\prime}=\partial_{0} f=-\partial_{\underline{x}} f$ similar to the complex case.

We also consider the hypercomplex monogenic polynomials in terms of the so-called Fueter variables

$$
z_{k}=x_{k}-x_{0} e_{k}, k=1, \ldots, n .
$$

This implies the use of the Fueter polynomials of degree of homogeneity $k$, as defined in [2]

$$
z_{1}^{v_{1}} \times \ldots \times z_{n}^{v_{n}}:=\frac{1}{k !} \sum_{\pi\left(i_{1}, \ldots, i_{n}\right)} z_{i_{1}} \cdots z_{i_{n}}
$$

where the sum is taken over all $k !=|v|$ ! permutations of $\left(i_{1}, \ldots, i_{n}\right), i_{j} \in\{1, \ldots, n\}, j=1, \ldots, n$, (see also [3]).

\section{HYPERCOMPLEX APPELL SEQUENCES}

We recall the classical definition of sequences of Appell polynomials [4] adapted to the hypercomplex case: a sequence of monogenic polynomials $\left(\mathscr{F}_{k}\right)_{k>0}$ of exact degree $k$ is called a generalized Appell sequence if $\mathscr{F}_{0}(x) \equiv 1$ and $\mathscr{F}_{k}^{\prime}=k \mathscr{F}_{k-1}, k=1,2, \ldots$ (see, e.g. [5]). The equivalent definition through the validity of a binomial-type expansion is provided by ([6]):

Theorem 1 A monogenic polynomial sequence $\left(\mathscr{F}_{k}\right)_{k \geq 0}$ is an Appell set if and only if it satisfies the binomial-type expansion

$\mathscr{F}_{k}(x)=\mathscr{F}_{k}\left(x_{0}+\underline{x}\right)=\sum_{s=0}^{k}\left(\begin{array}{l}k \\ s\end{array}\right) \mathscr{F}_{k-s}\left(x_{0}\right) \mathscr{F}_{s}(\underline{x}), x \in \mathscr{A}_{n}$. 
Intensively studied have been the so-called standard homogeneous Appell polynomials ( $[5,6,7])$ which can be written in the form

$$
\mathscr{P}_{k}\left(x_{0}, \underline{x}\right)=\sum_{s=0}^{k}\left(\begin{array}{l}
k \\
s
\end{array}\right) c_{s}(n) x_{0}^{k-s} \underline{x}^{s},
$$

where

$$
c_{s}(n)= \begin{cases}\frac{s ! !(n-2) ! !}{(n+s-1) ! !}, & \text { if } s \text { is odd, } \\ c_{s-1}(n), & \text { if } s \text { is even. }\end{cases}
$$

The study of sets of Appell polynomials has developed in several directions using different methods, see, e.g. [8, $9,10,6,11]$. For our purpose here, we call the attention to the following homogeneous monogenic polynomials

$$
\mathscr{R}_{i}^{k}(x)=\left(x_{0}+\left(i_{1} x_{1}+\cdots+i_{n} x_{n}\right)\left(i_{1} e_{1}+\cdots+i_{n} e_{n}\right)\right)^{k},
$$

where $\boldsymbol{i}:=\left(i_{1}, \ldots, i_{n}\right)$ is a real unit parameter-vector, i.e. $i_{1}^{2}+\cdots+i_{n}^{2}=1$. It follows immediately from this last condition that, since $\left(i_{1} e_{1}+\cdots+i_{n} e_{n}\right)^{2}=-1$, the polynomials (4) are isomorphic to the complex integer powers of $z$ and thereby they are called pseudo-complex polynomials. In this way, it is clear that $\left(\mathscr{R}_{i}^{k}\right)_{k>0}$ is a sequence of homogeneous monogenic paravector valued Appell polynomials.

Restricting ourselves from now on to the case $n=2$, the following result of [12] shows the importance of the aforementioned two types of Appell polynomials.

Theorem 2 There are exactly two different types of nontrivial Appell polynomials in $\mathbb{R}^{3}$, of the form

$$
\mathscr{P}_{k}\left(x_{0}, x_{1}, x_{2}\right)=\sum_{s=0}^{k}\left(\begin{array}{l}
k \\
s
\end{array}\right) d_{s} x_{0}^{k-s}\left(X_{1} e_{1}+X_{2} e_{2}\right)^{s}
$$

where $X_{j}=X_{j}\left(x_{1}, x_{2}\right), j=1,2$, are two real valued linear functions $\left(\partial_{1} X_{1} \cdot \partial_{2} X_{2} \neq 0\right)$ and $d_{0}=1$, namely

1. The 3D standard Appell polynomials (3),

$$
\mathscr{P}_{k}(x)=\sum_{s=0}^{k}\left(\begin{array}{l}
k \\
s
\end{array}\right) c_{s}(n) x_{0}^{k-s}\left(x_{1} e_{1}+x_{2} e_{2}\right)^{s},
$$

where

$$
c_{s}(n)=\left(\begin{array}{c}
s \\
\left\lfloor\frac{s}{2}\right\rfloor
\end{array}\right)
$$

and $\lfloor\cdot\rfloor$ is the floor function.

2. The 3D pseudo-complex polynomials (4),

$$
\mathscr{R}_{i}^{k}(x)=\left(x_{0}+\left(i_{1} x_{1}+i_{2} x_{2}\right)\left(i_{1} e_{1}+i_{2} e_{2}\right)\right)^{k},
$$

where $i_{1}^{2}+i_{2}^{2}=1$.
The use of the Fueter variables (1) allows to rewrite the $3 \mathrm{D}$ pseudo-complex polynomials in the form

$$
\mathscr{R}_{i}^{k}\left(z_{1}, \ldots, z_{n}\right)=\left(z_{1} i_{1}+\cdots+z_{n} i_{n}\right)^{k}\left(i_{1} e_{1}+\cdots+i_{n} e_{n}\right)^{k} .
$$

All this together with a result of [13, Theorem 1], concerned with the construction of a complete set of pseudocomplex Appell polynomials, allows to conclude the following:

Theorem 3 The set $\left\{\mathscr{R}_{i_{s}^{k}}^{k}\right\}_{s=0}^{k}$ is a basis for the space of homogeneous monogenic paravector valued polynomials of degree $k$ in $\mathbb{R}^{3}$, provided that the $k+1$ unit vectors $\boldsymbol{i}_{s}^{k}=\left(i_{s 1}^{k}, i_{s 2}^{k}\right) \in \mathbb{R}^{2}$, with $s=0, \ldots, k$, are pairwise noncollinear.

\section{TWO EXAMPLES OF A COMPLETE SET OF PSEUDO-COMPLEX POWERS}

The motivation for the present work came from the idea of developing an efficient algorithm to obtain pseudocomplex bases of Appell polynomials. The fact that, apart from the concrete parameter choice, the pseudocomplex polynomials $\mathscr{R}_{i}^{k}$ obey all the same principle structure, isomorphic to the complex powers $z^{k}$, suggested immediately the idea of economizing numerical costs for their construction. Moreover, and as we will see in this section, it leads us to an interesting study about the appropriate parameter choice and its connection with the primitive roots of unity. Other aspects of monogenic polynomials, for instance of combinatorial nature, are discussed in [14].

In this section we construct two particular bases of the form

$$
\left\{\mathscr{R}_{\boldsymbol{i}_{0}^{k}}^{k}, \mathscr{R}_{\boldsymbol{i}_{1}^{k}}^{k}, \ldots, \mathscr{R}_{\boldsymbol{i}_{k-1}^{k}}^{k}, \mathscr{R}_{\boldsymbol{i}_{k}^{k}}^{k}\right\}
$$

for the space of homogeneous monogenic paravector valued polynomials of degree $k$ in $\mathbb{R}^{3}$. It is clear that conditions referred in Theorem 3 still allow a wide choice of parameter-vectors, leading to different sets in (5). In fact, each unit vector $\boldsymbol{i}_{s}^{k}$ can be written in the form

$$
i_{s}^{k}=\left(\cos \alpha_{s}^{k}, \sin \alpha_{s}^{k}\right),
$$

where $\alpha_{s}^{k}$ are real numbers. The parameter set

$$
\mathbf{A}^{k}=\left\{\alpha_{0}^{k}, \alpha_{1}^{k}, \ldots, \alpha_{k}^{k}\right\}
$$

defines completely the set of unit vectors

$$
\left\{\boldsymbol{i}_{0}^{k}, \boldsymbol{i}_{1}^{k}, \ldots, \boldsymbol{i}_{k}^{k}\right\}
$$

and therefore, according to Theorem 3 , in order to obtain a basis, the parameter set (6) should be defined in such a way that the $k+1$ unit vectors in (7) are pairwise 
noncollinear. Throughout this work, a set (6) in the aforementioned conditions is called a primitive parameter set. We present now two different primitive parameter sets both connected, as it seems to be natural, with the primitive roots of unity.

Example 1 For $k=0,1, \ldots$, the set

$$
\mathbf{A}^{k}=\left\{\alpha_{s}^{k}=-\frac{\pi}{2}+(s+1) \frac{\pi}{k+1}, s=0, \ldots, k\right\},
$$

is obviously a primitive parameter set. This natural choice of the parameters $\alpha_{s}^{k}$ leads to a set of $k+1$ equally distributed points in the real interval ] $\left.-\frac{\pi}{2}, \frac{\pi}{2}\right]$ (see Table 1) which are associated with primitive roots of unity of a certain order.

TABLE 1. Primitive parameter sets associated with Example 1.

\begin{tabular}{ll}
$k$ & $\mathbf{A}^{k}$ \\
\hline 0 & $\left\{\frac{\pi}{2}\right\}$ \\
1 & $\left\{0, \frac{\pi}{2}\right\}$ \\
2 & $\left\{-\frac{\pi}{6}, \frac{\pi}{6}, \frac{\pi}{2}\right\}$ \\
3 & $\left\{-\frac{\pi}{4}, 0, \frac{\pi}{4}, \frac{\pi}{2}\right\}$ \\
4 & $\left\{-\frac{3 \pi}{10},-\frac{\pi}{10}, \frac{\pi}{10}, \frac{3 \pi}{10}, \frac{\pi}{2}\right\}$
\end{tabular}

The first pseudo-complex powers associated with the choice (8) of the primitive parameter set are:

- $\mathscr{R}_{i_{0}^{0}}^{0}=1$;

- $\mathscr{R}_{\boldsymbol{i}_{0}^{1}}^{1}=z_{1} e_{1}, \mathscr{R}_{\boldsymbol{i}_{1}^{1}}^{1}=z_{2} e_{2}$;

- $\mathscr{R}_{i_{0}^{2}}^{2}=-\frac{1}{4}\left(\sqrt{3} z_{1}-z_{2}\right)^{2}, \mathscr{R}_{i_{1}^{2}}^{2}=-\frac{1}{4}\left(\sqrt{3} z_{1}+z_{2}\right)^{2}$,

$$
\mathscr{R}_{i_{2}^{2}}^{2}=-z_{2}^{2}
$$

- $\mathscr{R}_{i_{0}^{3}}^{3}=-\frac{1}{4}\left(z_{1}-z_{2}\right)^{3}\left(e_{1}-e_{2}\right), \mathscr{R}_{i_{1}^{3}}^{3}=-z_{1}^{3} e_{1}$,

$$
\mathscr{R}_{i_{2}^{3}}^{3}=-\frac{1}{4}\left(z_{1}+z_{2}\right)^{3}\left(e_{1}+e_{2}\right), \mathscr{R}_{i_{3}^{3}}^{3}=-z_{2}^{3} e_{2} .
$$

We point out that for the choice (8), $\mathbf{A}^{k-1} \subset \mathbf{A}^{k^{\prime}-1}$ if and only if $k$ and $k^{\prime}$ are integer powers of 2, with $k<k^{\prime}$. This observation was the motivation for constructing another parameter set in such a way that $\mathbf{A}^{k} \subset \mathbf{A}^{k+1}$, i.e.

$$
\begin{aligned}
\mathbf{A}^{k+1} & =\left\{\alpha_{0}^{k+1}, \alpha_{1}^{k+1}, \ldots, \alpha_{k}^{k+1}, \alpha_{k+1}^{k+1}\right\} \\
& =\left\{\alpha_{0}^{k}, \alpha_{1}^{k}, \ldots, \alpha_{k}^{k}, \alpha_{k+1}^{k+1}\right\}=\mathbf{A}^{k} \cup\left\{\alpha_{k+1}^{k+1}\right\} .
\end{aligned}
$$

This idea was firstly considered in [14], where the following primitive parameter set plays an important role.

Example 2 The set

$$
\mathbf{A}^{k}=\left\{\alpha_{0}^{k}, \alpha_{1}^{k}, \ldots, \alpha_{k}^{k}\right\}
$$

with

$$
\begin{aligned}
& \alpha_{0}^{k}=\alpha_{0}^{0}=\frac{\pi}{2}, \\
& \alpha_{s}^{k}=\alpha_{s}^{s}=\frac{\pi}{2}-\frac{\left(2 n^{\prime}+1\right) \pi}{2^{n+1}}, \text { for } s=1, \ldots, k,
\end{aligned}
$$

where $n$ and $n^{\prime}$ are integers such that $s=2^{n}+n^{\prime}$ and $n^{\prime}=0, \ldots, 2^{n}-1$, is a primitive parameter set.

Table 2 contains the first parameter sets corresponding to the choice (10). In this table we highlight, for each degree $k$, the new element $\alpha_{k}^{k}$.

TABLE 2. Primitive parameter sets associated with Example 2.

\begin{tabular}{ll}
$k$ & $\mathbf{A}_{k}$ \\
\hline 0 & $\left\{\frac{\boldsymbol{\pi}}{\mathbf{2}}\right\}$ \\
1 & $\left\{\mathbf{0}, \frac{\pi}{2}\right\}$ \\
2 & $\left\{0, \frac{\pi}{\mathbf{4}}, \frac{\pi}{2}\right\}$ \\
3 & $\left\{-\frac{\pi}{\mathbf{4}}, 0, \frac{\pi}{4}, \frac{\pi}{2}\right\}$ \\
4 & $\left\{-\frac{\pi}{4}, 0, \frac{\pi}{4}, \frac{\mathbf{3 \pi}}{\mathbf{8}}, \frac{\pi}{2}\right\}$ \\
5 & $\left\{-\frac{\pi}{4}, 0, \frac{\pi}{8}, \frac{\pi}{4}, \frac{3 \pi}{8}, \frac{\pi}{2}, \frac{3 \pi}{8}\right\}$ \\
6 & $\left\{-\frac{\pi}{8},-\frac{\pi}{4}, 0, \frac{\pi}{8}, \frac{\pi}{4}, \frac{3 \pi}{8}, \frac{\pi}{2}, \frac{3 \pi}{8}\right\}$ \\
7 & $\left\{-\frac{3 \pi}{8},-\frac{\pi}{8},-\frac{\pi}{4}, 0, \frac{\pi}{8}, \frac{\pi}{4}, \frac{3 \pi}{8}, \frac{\pi}{2}, \frac{3 \pi}{8}\right\}$
\end{tabular}

By construction, $\mathbf{A}^{k}$ satisfies (9) and it is easy to see that $\mathbf{A}^{k}$ can be written explicitly as

$$
\mathbf{A}^{k}=\left\{\frac{l \pi}{2^{n}}\right\}_{l=-2^{n-1}+1}^{2^{n-1}} \cup\left\{\frac{\pi}{2}-\frac{(2 l+1) \pi}{2^{n+1}}\right\}_{l=0}^{n^{\prime}} .
$$

Last expression reveals a block structure (see Figure 2) which:

- for $k=2^{n}-1$, reduces to all the $2^{n}$ equally distributed points referred in Example 1;

- for $k=2^{n}, 2^{n}+1, \ldots, 2^{n+1}-1$, adds to all the $2^{n}$ elements of $\mathbf{A}^{2^{n}-1}$ the correspondent odd multiples of $\frac{\pi}{2^{n+1}}$ in $\left.]-\frac{\pi}{2}, \frac{\pi}{2}\right]$.

The first pseudo-complex powers corresponding to the primitive parameter set (10) are as follows:

- $\mathscr{R}_{i_{0}^{0}}^{0}=1$;

- $\mathscr{R}_{i_{0}^{1}}^{1}=z_{2} e_{2}, \quad \mathscr{R}_{i_{1}^{1}}^{1}=z_{1} e_{1}$

- $\quad \mathscr{R}_{i_{0}^{2}}^{2}=-z_{2}^{2}, \quad \mathscr{R}_{i_{1}^{2}}^{2}=-z_{1}^{2}, \quad \mathscr{R}_{\boldsymbol{i}_{2}^{2}}^{2}=-\frac{1}{2}\left(z_{1}+z_{2}\right)^{2}$;

- $\mathscr{R}_{i_{0}^{3}}^{3}=-z_{2}^{3} e_{2}, \quad \mathscr{R}_{i_{1}^{3}}^{3}=-z_{1}^{3} e_{1}$,

$\mathscr{R}_{i_{2}^{3}}^{3}=-\frac{1}{4}\left(z_{1}+z_{2}\right)^{3}\left(e_{1}+e_{2}\right)$,

$\mathscr{R}_{i_{3}^{3}}^{3}=-\frac{1}{4}\left(z_{1}-z_{2}\right)^{3}\left(e_{1}-e_{2}\right)$. 

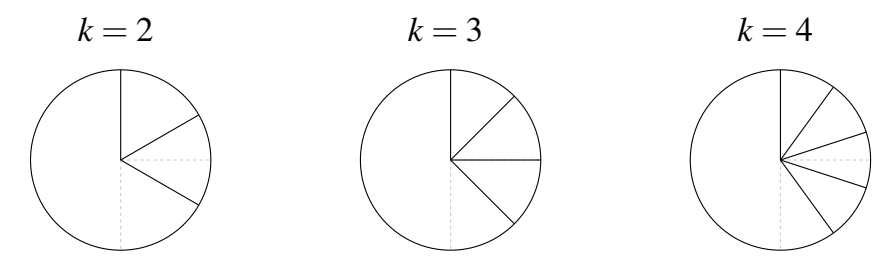

FIGURE 1. Primitive parameter sets of Example 1.

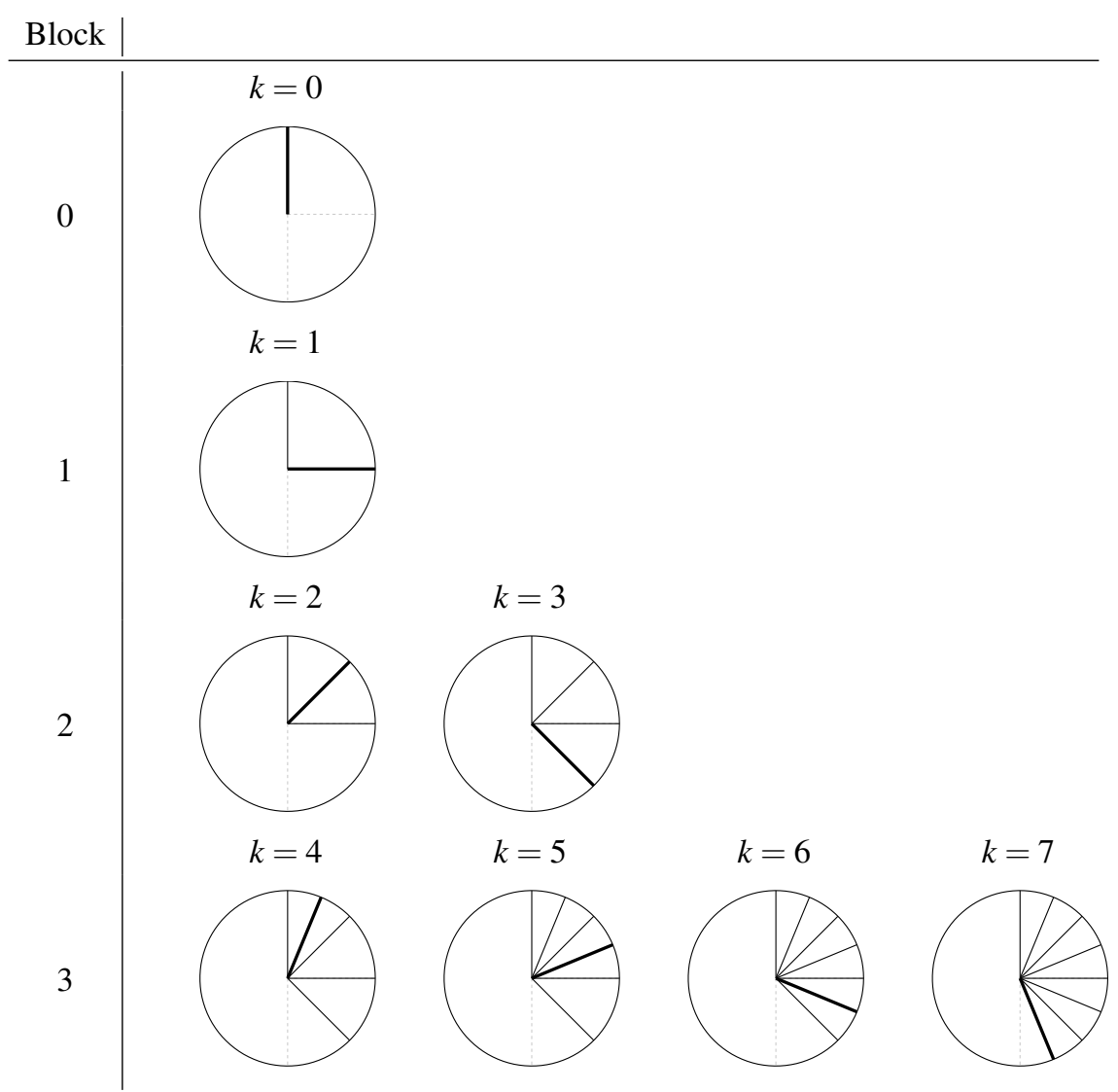

FIGURE 2. The block structure of the primitive parameter sets of Example 2.

\section{COMPUTATIONAL CONSIDERATIONS}

In [14] the parameter set (10) was used to obtain a link between combinatorics and hypercomplex function theory, by means of a new combinatorial identity. Our objective here is rather different, as we intent to study pseudo-complex polynomials associated with the choice (10) in a more detailed way and to stress their numerical costs advantages compared with the Fueter polynomials.

Needless to say that the aforementioned parameter set has obvious advantages from the computational point of view, when we compare it with the parameter set (8). In fact, denoting by $\mathfrak{I}_{s}^{k}$ the unit vector

$$
\mathfrak{I}_{s}^{k}:=\cos \alpha_{s}^{k} e_{1}+\sin \alpha_{s}^{k} e_{2},
$$

then it is clear that (10b) implies that

$$
\boldsymbol{i}_{s}^{k}=\boldsymbol{i}_{s}^{s} \quad \text { and } \quad \mathfrak{I}_{s}^{k}=\mathfrak{I}_{s}^{s}, s=0, \ldots, k .
$$

Therefore, using for simplicity, $R_{s}^{k}:=\mathscr{R}_{i_{s}^{s}}^{k}$ and $I_{s}:=\mathfrak{I}_{s}^{s}$, we can write, for $s=0, \ldots, k$,

$$
\begin{aligned}
R_{s}^{k} & =\left(x_{0}+\left(\cos \alpha_{s}^{k} x_{1}+\sin \alpha_{s}^{k} x_{2}\right) \mathfrak{I}_{s}^{k}\right)^{k} \\
& =\left(x_{0}+\left(\cos \alpha_{s}^{s} x_{1}+\sin \alpha_{s}^{s} x_{2}\right) I_{s}\right)^{k},
\end{aligned}
$$

which means that

$$
R_{s}^{k}=R_{s}^{k-1} R_{s}^{1},
$$

for $s=0, \ldots, k-1$, and

$$
R_{k}^{k}=\left(R_{k}^{1}\right)^{k}
$$


Here we use also the simplified notation $R_{s}^{1}$ for denoting the first degree polynomial $\mathscr{R}_{\boldsymbol{i}_{s}^{s}}^{1}$.

In other words, the first $k-1$ pseudo-complex polynomials (PCP) of degree $k$ can be obtained by simply multiplying the polynomials of degree $k-1$ by an appropriated first degree polynomial (see Figure 3).

In this way, the first pseudo-complex powers corresponding to the primitive parameter set (10) can be obtained in the following way:

- $R_{0}^{0}=1$;

- $R_{0}^{1}=x_{0}+x_{2} I_{0}, \quad R_{1}^{1}=x_{0}+x_{1} I_{1} ;$

- $R_{0}^{2}=R_{0}^{1} R_{0}^{1}, \quad R_{1}^{2}=R_{1}^{1} R_{1}^{1}$, $R_{2}^{2}=\left(R_{2}^{1}\right)^{2}$, where $R_{2}^{1}=x_{0}+\frac{\sqrt{2}}{2}\left(x_{1}+x_{2}\right) I_{2}$;

- $R_{0}^{3}=R_{0}^{2} R_{0}^{1}, \quad R_{1}^{3}=R_{1}^{2} R_{1}^{1}, \quad R_{2}^{3}=R_{2}^{2} R_{2}^{1}$, $R_{3}^{3}=\left(R_{3}^{1}\right)^{3}$, where $R_{3}^{1}=x_{0}+\frac{\sqrt{2}}{2}\left(x_{1}-x_{2}\right) I_{3}$.

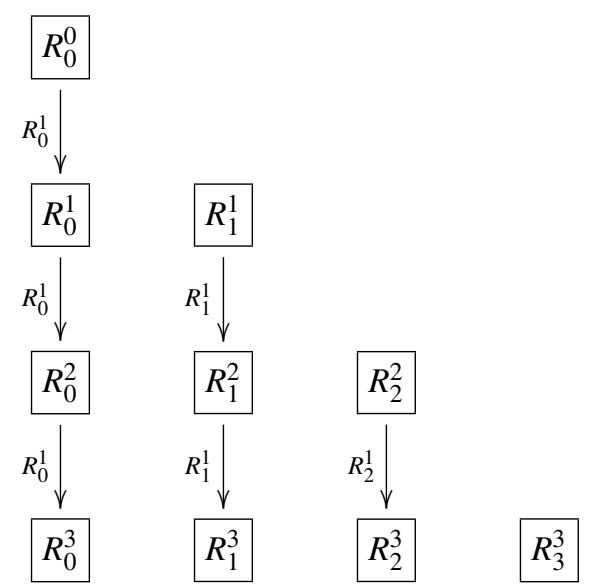

FIGURE 3. An efficient scheme to obtain a basis of PCP.

To continue our goal we recall now the well known fact that the generalized powers (2) form a paravector valued basis for the Taylor series of a monogenic function, which is often used for practical applications (see, e.g. [15]). Moreover, as is indicated in [2], the polynomials $F_{s}^{k}:=z_{1}{ }^{k-s} \times z_{2}{ }^{s}$ satisfy the recursion formula

$$
F_{s}^{k}=\frac{1}{k}\left\{(k-s) z_{1} F_{s}^{k-1}+s z_{2} F_{s-1}^{k-1}\right\},
$$

for $s=1, \ldots, k-1$, with

$$
F_{0}^{k}=z_{1}^{k} \quad \text { and } \quad F_{k}^{k}=z_{2}^{k} .
$$

In order to compare the numerical costs of constructing a basis formed by the pseudo-complex polynomials with that one formed by the Fueter polynomials, it is enough to compute the number of basic operations associated to the schemes (11) and (12), respectively. In other words, to get an idea of how much it costs to obtain a basis of degree $k$, assuming that the corresponding basis of degree $k-1$ is known, let us count the number of multiplications required by both algorithms.

There are $4 k$ real multiplications in (11a). In addition, the use of $(11 \mathrm{~b})$ requires $4(k-1)$ multiplications. Hence, the total number of multiplications in this scheme is $8 k-4$.

On the other hand the use of the recursion formula (12a) involves $22(k-1)$ multiplications, while (12b) demands 8 operations. Therefore, this scheme requires the total of $22 k-14$ multiplications.

Roughly speaking, the above numbers illustrate the difference between multiplying two complex numbers and two paravectors. Of course that some care must be taken in comparing two algorithms of the same order. In particular, we have to be aware of the fact that (11b) involves also the computation of $\alpha_{k}^{k}$ and this in turn implies the evaluation of two trigonometric functions.

Our main objective was to study a certain type of Appell polynomials with properties similar to the complex powers and to point out the computational efficiency of the algorithm developed for the construction of a basis for the space of homogeneous monogenic paravector valued polynomials in $\mathbb{R}^{3}$.

\section{ACKNOWLEDGMENTS}

This work was supported by FEDER founds through COMPETE-Operational Programme Factors of Competitiveness ("Programa Operacional Factores de Competitividade") and by Portuguese funds through the Center for Research and Development in Mathematics and Applications (University of Aveiro) and the Portuguese Foundation for Science and Technology ("FCT-Fundação para a Ciência e a Tecnologia"), within project PEst-C/MAT/UI4106/2011 with COMPETE number FCOMP-01-0124-FEDER-022690. The research of the first author was also supported by FCT under the fellowship SFRH/BD/44999/2008.

\section{REFERENCES}

1. K. Gürlebeck, and H. Malonek, Complex Variables Theory Appl. 39, 199-228 (1999).

2. K. Gürlebeck, K. Habetha, and W. Sprößig, Holomorphic functions in the plane and n-dimensional space, Birkhäuser Verlag, Basel, 2008.

3. H. Malonek, Complex Variables 14, 25-33 (1990).

4. P. Appell, Ann. Sci. École Norm. Sup. 9, 119-144 (1880).

5. M. I. Falcão, and H. Malonek, "Generalized exponentials through Appell sets in $\mathbb{R}^{n+1}$ and Bessel functions," in AIP Conference Proceedings, edited by T. E. Simos, 
G. Psihoyios, and C. Tsitouras, 2007, vol. 936, pp. 738-741.

6. I. Cação, M. I. Falcão, and H. Malonek, Math. Comput. Model. 53, 1084-1094 (2011).

7. I. Cação, M. I. Falcão, and H. Malonek, Comput. Methods Funct. Theory 12, 371-391 (2012).

8. H. R. Malonek, and R. De Almeida, Appl. Math. Lett. 23, 1174-1178 (2010).

9. S. Bock, and K. Gürlebeck, Math. Methods Appl. Sci. 33, 394-411 (2010).

10. R. Lávička, Archivum Mathematicum 46, 339-349 (2010).

11. I. Cação, M. I. Falcão, and H. Malonek, Lecture Notes in Computer Science, 6784, 271-286 (2011).

12. H. Malonek, and M. I. Falcão, Advances in Applied Clifford Algebras, (2012) doi:10.1007/s00006-012-03615.

13. I. Cação, and H. Malonek, "On Complete Sets of Hypercomplex Appell Polynomials," in AIP Conference Proceedings, edited by T. E. Simos, G. Psihoyios, and C. Tsitouras, 2008, vol. 1048, pp. 647-650.

14. C. Cruz, M. I. Falcão, and H. Malonek, "On the role of the central binomial coefficients in generalized Appell polynomials of hypercomplex variables", submitted for publication (2012).

15. S. Bock, M. I. Falcão, K. Gürlebeck, and H. Malonek, Journal of Computational and Applied Mathematics 189, 67-79 (2006). 